\title{
Netrin-1 promotes retinoblastoma-associated angiogenesis
}

\author{
Xiaosheng Yang ${ }^{1 \#}$, Hui Sun ${ }^{2 \#}$, Tianchi Tang ${ }^{3}$, Wenchuan Zhang ${ }^{1}$, Yi Li ${ }^{1}$ \\ ${ }^{1}$ Department of Neurosurgery, Shanghai Ninth People's Hospital, Shanghai Jiao Tong University School of Medicine, Shanghai, China; ${ }^{2}$ Department \\ of Neurosurgery, Xinhua Hospital, Shanghai Jiao Tong University School of Medicine, Shanghai, China; ${ }^{3}$ Department of Neurosurgery, Second \\ Affiliated Hospital, School of Medicine, Zhejiang University, Hangzhou, China \\ Contributions: (I) Conception and design: X Yang; (II) Administrative support: X Yang; (III) Provision of study materials or patients: X Yang; (IV) \\ Collection and assembly of data: All authors; (V) Data analysis and interpretation: All authors; (VI) Manuscript writing: All authors; (VII) Final \\ approval of manuscript: All authors. \\ "These authors contributed equally to this work. \\ Correspondence to: Wenchuan Zhang; Yi Li. Department of Neurosurgery, Shanghai Ninth People's Hospital, Shanghai Jiao Tong University School \\ of Medicine, 639 Zhizaoju Road, Shanghai 200011, China. Email: zhangwench88@sjtu.edu.cn; snailliyi@163.com.
}

Background: Retinoblastoma $(\mathrm{Rb})$ is the most common intraocular cancer of infancy and childhood, with an incidence of nearly $0.006 \%$ in all live births. Although a functional loss or inactivation of both alleles of the retinoblastoma 1 (RB1) gene during retinal development appears to be the predominant etiology for Rb, genes associated with tumor angiogenesis are also likely to be involved in the development of this condition. Netrin-1 is a factor that regulates pathological angiogenesis, while its role in Rb is largely unknown. The present study examined the role of netrin-1 in Rb.

Methods: The expression of netrin-1 in Rb was assessed using public databases and using clinical specimens by RT-qPCR for mRNA and by ELISA for protein. The expression of netrin-1 was suppressed in Rb by siRNA and the effects on cell growth were determined by a CCK-8 assay, while the effects on angiogenesis were examined in vitro using human umbilical vein endothelial cell (HUVEC) assays and in vivo by quantification of tumor vessel density.

Results: Analysis of published databases revealed that the netrin-1 gene is significantly upregulated in $\mathrm{Rb}$, which was confirmed by immunohistochemistry on clinical specimens. Inhibition of netrin-1 in Rb cell lines significantly reduced their effects on angiogenesis in vitro using a HUVEC co-culture assay without affecting cell growth. Inhibition of netrin-1 expression in vivo suppressed the growth of grafted Rb, and this effect could be abolished by co-expression of vascular endothelial growth factor A (VEGF-A).

Conclusions: This data demonstrated a novel role for netrin-1 in the regulation of $\mathrm{Rb}$-associated cancer vascularization and may represent a novel therapeutic target for patients with $\mathrm{Rb}$.

Keywords: Retinoblastoma (Rb); netrin-1; angiogenesis; vascular endothelial growth factor A (VEGF-A)

Submitted Sep 22, 2021. Accepted for publication Nov 10, 2021.

doi: $10.21037 / \mathrm{atm}-21-5560$

View this article at: https://dx.doi.org/10.21037/atm-21-5560

\section{Introduction}

Retinoblastoma $(\mathrm{Rb})$ is the most common intraocular cancer of infancy and childhood, with an incidence of nearly $0.006 \%$ in all live births (1). A functional loss or inactivation of both alleles of the retinoblastoma 1 ( $R B 1)$ gene during retinal development is believed to be the predominant etiology for $\mathrm{Rb}$ (2). However, additional genetic changes are required for the continued growth and spread of Rb. Specifically, genes associated with tumor angiogenesis are likely to be involved in the development and progression of this cancer (3).

Unfortunately, current treatments for $\mathrm{Rb}$ have been hampered by an incomplete understanding of the specific pathogenesis of the disease and thus, a lack of a specific molecular target. Earlier studies have focused on blood supply as a potential therapeutic target (3). The 
histopathology of $\mathrm{Rb}$ has demonstrated the presence of viable tumor cells adjacent to blood vessels surrounded by necrotic cells, which graphically illustrates the exquisite dependence of $\mathrm{Rb}$ cells on blood supply (3). Indeed, the outgrowth of $\mathrm{Rb}$ relies on adequate angiogenesis, which is accomplished through expression of proangiogenic factors such as vascular endothelial growth factor A (VEGF-A) (4). Inhibition of angiogenesis has been shown to effectively eliminate $\mathrm{Rb}$ cells $(5,6)$, suggesting that anti-angiogenic therapy may be a promising innovative treatment strategy against this specific vulnerability of the cancer. However, VEGF-A is a potent proangiogenic factor and plays a substantial role in the homeostasis of various tissues and organs in the human body (7-11) and therefore, therapies targeting VEGF-A may cause a multitude of adverse side effects. Thus, an alternative angiogenic target must be sought.

Netrin-1 is a diffusible, laminin-associated protein that acts as a guidance cue during neurogenesis (12). During development, netrin-1 is predominantly expressed in the central nervous system (12) while in adult mammals, netrin-1 and its receptors are expressed in both neural and non-neural tissues (13). Interestingly, netrin-1 has been found to be highly expressed in human metastatic breast tumors (14) and aggressive neuroblastoma (15) and is likely related to tumor-associated angiogenesis (16). Moreover, netrin-1 has been shown to regulate angiogenesis in diabetic kidney disease (17). However, the precise role of netrin-1 in Rb has not been fully examined. This current study investigated the expression and role of netrin- 1 in $\mathrm{Rb}$ both in vitro and in vivo. We present the following article in accordance with the ARRIVE reporting checklist (available at https://dx.doi.org/10.21037/atm-21-5560).

\section{Methods}

\section{Culture and transfection of human Rb cell lines}

Human Rb cell lines Y79, WERI-Rb-1, NCC-Rbc-57, NCC-Rbc-92, and NCC-Rbc-T1 were purchased from American Type Culture Collection (ATCC, Rockville, MD, USA) and Creative Bioarray (Shirley, NY, USA). All cells were maintained in Dulbecco's modified Eagle's medium (DMEM, Invitrogen, St. Louis, MO, USA) supplemented with L-glutamine and 5\% fetal bovine serum (FBS; SigmaAldrich, St Louis, MO, USA) in a humidified chamber with $5 \% \mathrm{CO}_{2}$ at $37^{\circ} \mathrm{C}$. Plasmids expressing small interfering (si)RNA netrin-1 or a scrambled sequence for netrin-1 together with a luciferase reporter (to allow in vivo cell tracing by bioluminescent assay) under a CMV promoter were purchased from Santa Cruz (SC-42044, Beijing, China). Transfections were performed with $1.5 \mu \mathrm{g}$ plasmid using Lipofectamine 3000 (Invitrogen).

\section{Animal experiments}

All experiments were performed under a project license (SH9H-2019-A529-1) granted by the institutional ethics board of the Shanghai Jiao Tong University, in compliance with institutional guidelines for the care and use of animals. A protocol was prepared before the study without registration. Male and female 12-week-old nude mice (body weight $\sim 22 \mathrm{~g}$; both genders were used to exclude a possible effect of sex on experimental results) were purchased from Shanghai Laboratory Animal Center (SLAC) (Shanghai, China). Both male and female nude mice were used and distributed evenly in each experimental group. Mice were housed under a 12-hour light-dark cycle. A power test $(\mathrm{P}<0.05)$ was performed to determine the exact number of mice to obtain legitimate effects. Grouping included an allocation concealment method to ensure minimal confounders. No criteria were used for excluding animals (or experimental units) during the experiment, and no data were excluded during the analysis. The study did not have humane endpoints.

For xenograft procedures and bioluminescence analyses, mice were anesthetized by $2.5 \%$ isoflurane inhalation. Transfected WERI-Rb-1 cells were subcutaneously grafted into the mice (200 cells/mouse). The xeno-tumor was assessed with a bioluminescent assay (IVIS imaging system, Perkin Elmer, Santa Clara, CA, USA) 1 month after transplantation. The mice were assigned into the following three groups, and each group contained 5 mice. Group 1 mice received subcutaneous transplantation of control WERI-Rb-1 cells transfected with a scrambled vector. Group 2 mice received subcutaneous transplantation of WERI-Rb-1 cells transfected with si-netrin-1 and group 3 mice received subcutaneous transplantation of WERIRb-1 cells transfected with si-netrin-1 and biweekly injections of recombinant VEGF-A (100 ng/mouse, SigmaAldrich) into the tumor site.

\section{Cell proliferation assay}

The in vitro proliferation potential of Rb cells was assessed with using the Cell Counting Kit-8 (CCK-8) assay (Roche, Indianapolis, IN, USA), in which the absorbance value (OD) 
of the cells was quantified at $540 \mathrm{~nm}$.

\section{Immunobistochemistry, enzyme-linked immunosorbent assay (ELISA), and quantification of vessel density}

Immunohistochemistry was performed using primary rabbit anti-netrin-1 (Abcam, Cambridge, MA, USA) and antiCD31 antibodies (Becton-Dickinson Biosciences, San Jose, CA, USA), followed by a secondary cy3-conjugated antirabbit antibody (Jackson ImmunoResearch Labs, West Grove, PA, USA). The quantification of the $\mathrm{CD} 31^{+}$area was performed using NIH ImageJ software, based on 5 slides that were $20 \mu \mathrm{m}$ apart from each other. Five mice were analyzed in each group. ELISA for netrin-1 was performed with a human Netrin-1 ELISA kit (ABIN6958077, antibodiesonline.com) according to the manufacturer's instructions. The absorption was measured at $450 \mathrm{~nm}$. The protein concentration was determined by comparing the relative absorbance of the samples with the standards.

\section{Real time quantitative polymerase chain reaction (RT-qPCR)}

A SYBR Green PCR Kit (Qiagen, Shanghai, China) was used for RT-qPCR with the designed primers purchased from Qiagen (no sequence information was provided). The RT-qPCR reactions were performed in triplicate. The gene values were assessed with the $2^{-\Delta \Delta C t}$ method and obtained after sequential normalization to $\beta$-actin as the experimental control.

\section{Bioinformatics and statistical analysis}

Data were obtained from the Gene Expression Omnibus (GEO, https://www.ncbi.nlm.nih.gov/geo/) public database (18). Two gene expression profiles (GSE174200 and GSE172170) were selected and the GEO2R online analysis tool was used to detect the differentially expressed genes (DEGs). The study was conducted in accordance with the Declaration of Helsinki (as revised in 2013). The $\mathrm{P}$ value, adjusted $\mathrm{P}$ value, and $\log$ fold change (FC) were calculated. Pathway enrichment analyses of the DEGs were performed using Metascape (https://metascape.org) and Kyoto Encyclopedia of Genes and Genomes (KEGG) (19). All data in the current study were summarized and statistically analyzed with GraphPad Prism 7 (GraphPad, Chicago, IL, USA). One-way analysis of variance (ANOVA) was performed to compare the data from different groups. The values are expressed as mean \pm standard deviation (SD).
A P value $<0.05$ was considered statistically significant.

\section{Results}

\section{Enhanced angiogenesis and upregulation of netrin-1 in $R b$}

Previous reports have suggested the importance of angiogenesis in the development and progression of $\mathrm{Rb}$. Thus, the angiogenesis status and the levels of netrin-1 were examined in $\mathrm{Rb}$ specimens. Data obtained from the GEO database were screened to identify the DEGs between $\mathrm{Rb}$ samples and control retina samples. Analyses of the significantly downregulated and upregulated genes revealed that they were enriched in many pathways including angiogenesis (Figure 1A). Moreover, netrin-1 was identified as a significantly upregulated gene in $\mathrm{Rb}$ samples (Figure $1 B$ ). These data suggested the $\mathrm{Rb}$ patients showed enhanced angiogenesis and upregulated netrin-1 expression.

\section{Enbanced netrin-1 expression in $R b$ and $R b$ cell lines}

Immunohistochemistry revealed significantly higher expression of netrin- 1 in $\mathrm{Rb}$ specimens compared to normal retina (Figure $2 A$ ). Moreover, higher levels of netrin-1 mRNA (Figure 2B) and protein (Figure 2C) expression were detected in $5 \mathrm{Rb}$ cell lines, named Y79, WERI-Rb-1, NCCRbc-57, NCC-Rbc-92, and NCC-Rbc-T1 compared to healthy retina samples. These data are consistent with the analyses of the GEO database samples, demonstrating an upregulation of netrin- 1 in $\mathrm{Rb}$.

\section{Silencing netrin-1 expression in cells}

To assess the functionality of netrin-1 in Rb, plasmids expressing the siRNA for netrin-1 were constructed with a luciferase reporter under a CMV promoter. A control plasmid carrying a scrambled sequence of netrin-1 was also constructed (Figure 3A). The knockdown efficiency of these plasmids was assessed in $5 \mathrm{Rb}$ cell lines using the luciferase assay. A significant depletion of both netrin-1 mRNA (Figure 3B) and protein (Figure 3C) expression was observed, suggesting that the si-netrin-1 effectively depleted netrin-1 expression in $\mathrm{Rb}$ cells.

\section{Netrin-1 depletion compromises angiogenesis without affecting $R \boldsymbol{b}$ growth in vitro}

To assess the effects of netrin-1 depletion on $\mathrm{Rb}$ cell growth, 

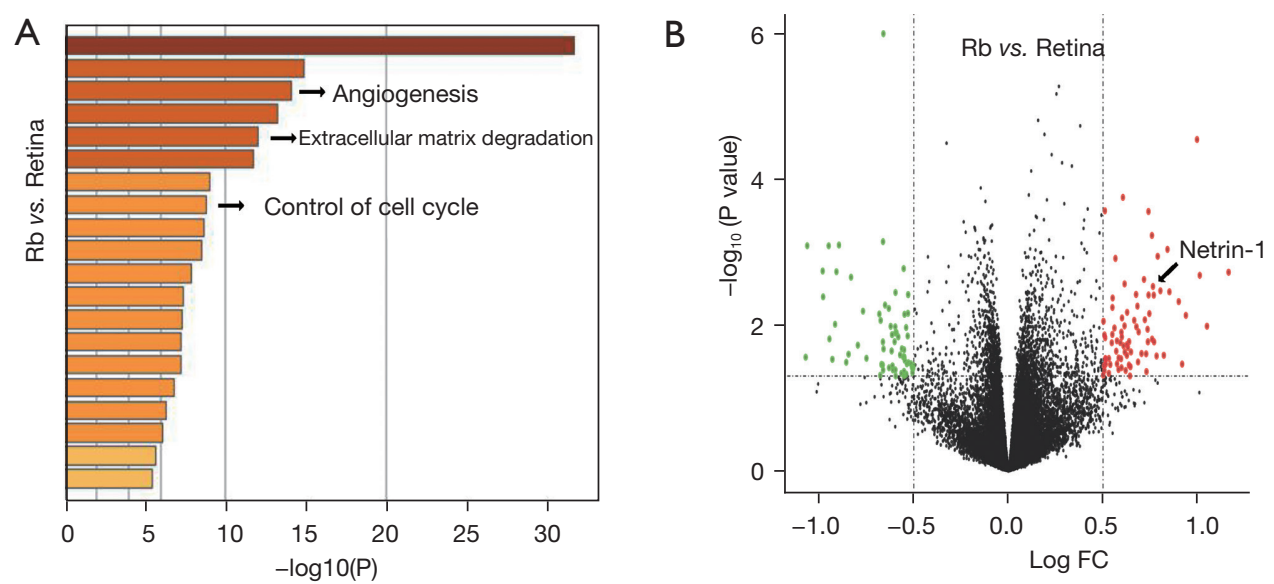

Figure $1 \mathrm{Rb}$ samples show enhanced angiogenesis and upregulation of netrin-1 compared to healthy retina samples. Data was obtained from the GEO database and analyzed using the online tool GEO2R. (A) The differentially expressed genes in Rb were enriched in numerous pathways including angiogenesis; (B) a volcano map of the differentially expressed genes in Rb samples shows netrin-1 as an upregulated gene.
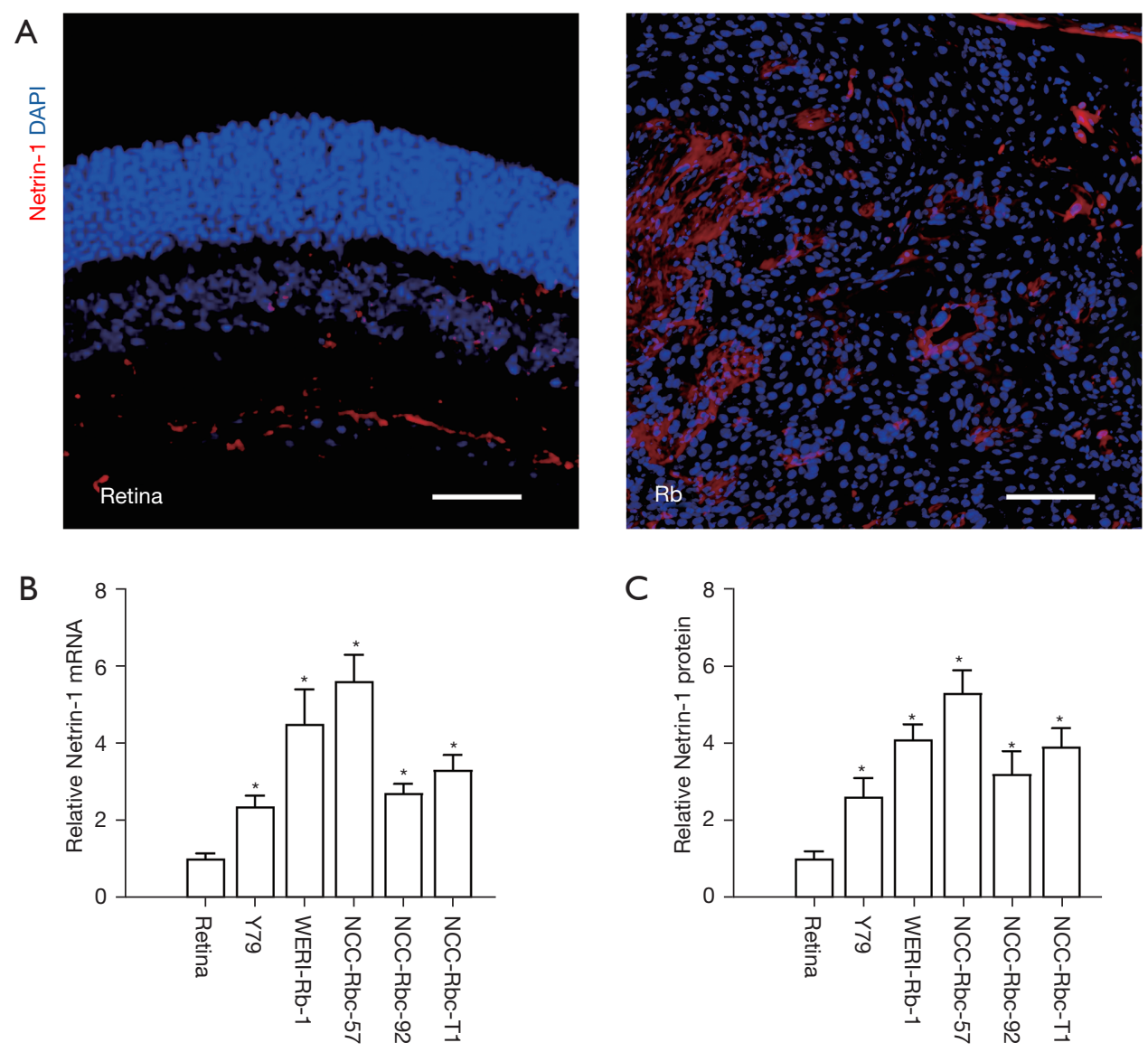

Figure 2 Netrin-1 expression is increased in $\mathrm{Rb}$ and $\mathrm{Rb}$ cell lines. (A) Representative immunohistochemistry images showing netrin-1 expression in Rb samples and normal retina samples; (B) relative netrin-1 mRNA expression in 5 Rb cell lines compared to a normal retina sample; (C) relative netrin-1 protein expression in $5 \mathrm{Rb}$ cell lines compared to a normal retina sample. Rb cells lines: $\mathrm{Y} 79$, WERI-Rb-1, NCC-Rbc-57, NCC-Rbc-92, and NCC-Rbc-T1; *, P<0.05; N=5; scale bar is $100 \mu \mathrm{m}$. 


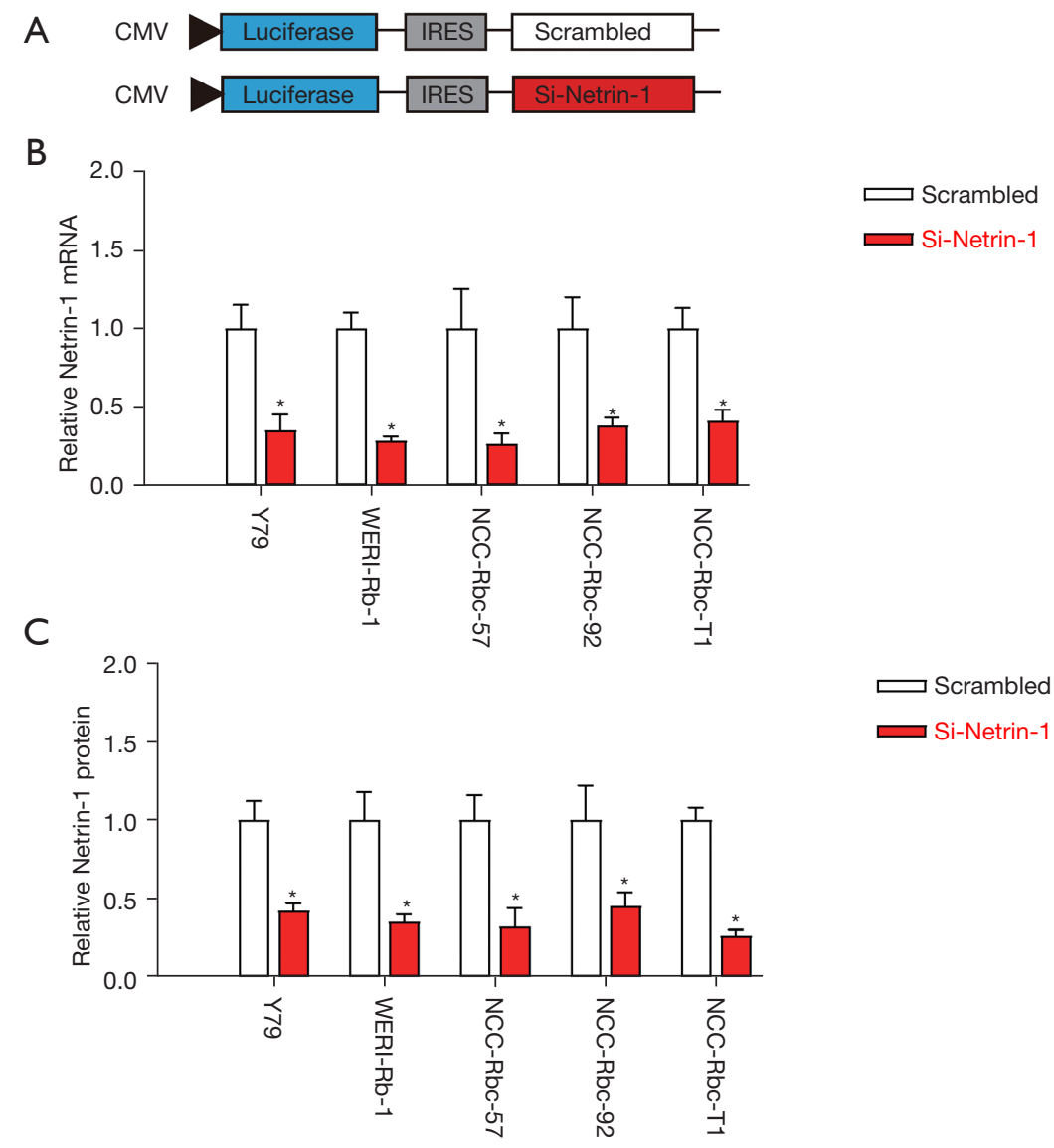

Figure 3 Silencing netrin-1 by construction of a siRNA plasmid. (A) Illustration of plasmids carrying the siRNA for netrin-1 or a scrambled netrin-1 sequence and a luciferase reporter under a CMV promoter; (B) relative netrin-1 mRNA expression in Rb cells transfected with sinetrin-1 or scrambled plasmid as assessed by RT-qPCR; (C) relative netrin-1 protein expression in Rb cells transfected with si-netrin-1 or scrambled plasmid as assessed by ELISA. Rb cell lines: Y79, WERI-Rb-1, NCC-Rbc-57, NCC-Rbc-92, and NCC-Rbc-T1; *, P<0.05; N=5. IRES, internal ribosome entry site; CMV, cytomegalovirus; RT-qPCR, 1uantitative reverse transcription polymerase chain reaction; ELISA, enzyme-linked immunosorbent assay.

CCK-8 assays were performed. Silencing of netrin-1 did not alter cell growth in either Y79 nor WERI-Rb-1 cells (Figure $4 A, 4 B$ ). When the si-netrin-1 transfected $\mathrm{Rb}$ cells were co-cultured with human umbilical vein endothelial cells (HUVECs) (Figure 4C), the vessel structure formation was significantly reduced (Figure $4 D, 4 E$ ), suggesting that netrin-1 depletion compromised angiogenesis without affecting $\mathrm{Rb}$ proliferation.

\section{Silencing netrin-1 compromises $R$ browth and tumor angiogenesis in vivo}

The effects of netrin-1 silencing on $\mathrm{Rb}$ growth was assessed in vivo using nude mice. Mice were given subcutaneous transplantation of WERI-Rb-1 cells transfected with either scrambled or si-netrin-1. Interestingly, netrin-1 depletion significantly reduced $\mathrm{Rb}$ growth in vivo (Figure $5 A, 5 B$ ), and this effect was abolished by administration of VEGF-A (Figure 5A,5B). Furthermore, silencing netrin-1 resulted in significantly reduced $\mathrm{Rb}$ tumor vessel density (Figure 5C,5D), and again, this effect was ameliorated in mice given VEGF-A (Figure 5C,5D). These results suggested that netrin-1 is required for $\mathrm{Rb}$-associated angiogenesis in cancer growth.

\section{Discussion}

Netrin-1 acts as a crucial guidance cue for axonal 

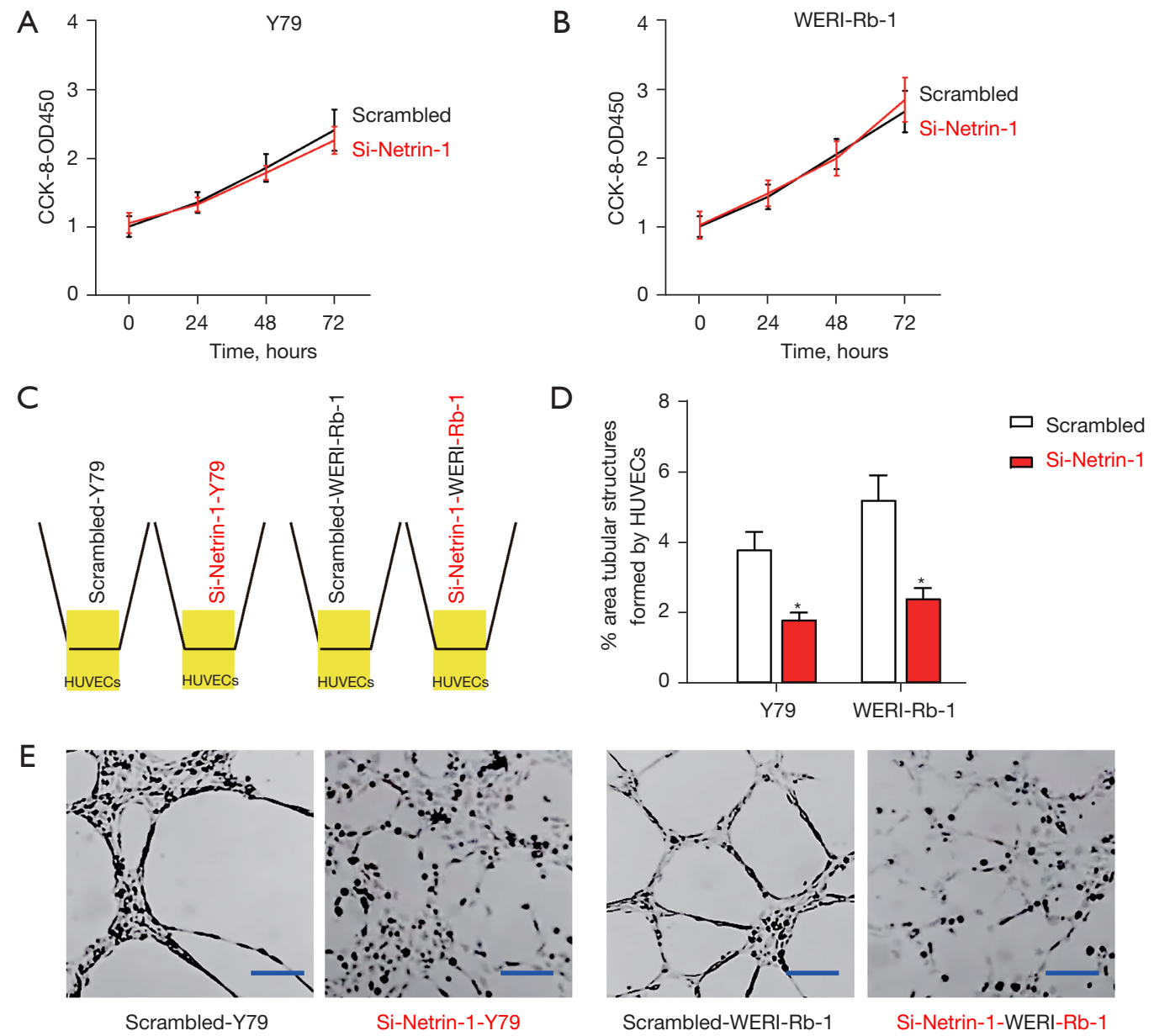

Figure 4 Netrin-1 depletion compromises angiogenesis without affecting Rb growth in vitro. (A,B) The CCK- 8 assay was used to assess cell proliferation in Rb cells transfected with si-netrin-1 or the scrambled plasmid; (C) transfected Rb cells were co-cultured with HUVECs as shown in the illustration; $(\mathrm{D}, \mathrm{E})$ the vessel structure formation was analyzed in both Y79 and WERI-Rb-1 cell lines. *, P<0.05; N=5; scale bar represents $100 \mu \mathrm{m}$. CCK-8, Cell Counting Kit-8; Rb, retinoblastoma; HUVEC, human umbilical vein endothelial cell; HUVECs, human umbilical vein endothelial cells.

growth cones and neurons, which is highly receptor dependent (12). Moreover, its angiogenesis capability has significant impact on tissue and organ development (12). Indeed, the current study demonstrated a proangiogenic role for netrin-1 in $\mathrm{Rb}$ growth and progression. This finding is important since the involvement of netrin- 1 in angiogenesis appears to be tissue- and organ- dependent. While some studies have shown that netrin-1 promotes angiogenesis (20), others have demonstrated an antiangiogenic effect (21). Furthermore, there is evidence to show that netrin-1 exhibits a proangiogenic effect at low concentrations but an antiangiogenic function in high concentrations (22). Thus, future studies should examine the effects of different levels of netrin-1 expression on $\mathrm{Rb}$ development.
This study demonstrated that netrin-1 may be a potential therapeutic target in $\mathrm{Rb}$. Targeting netrin-1, rather than the more potent proangiogenic factor VEGF-A, has several advantages. VEGF-A exerts many physiological and pathological effects on different tissues and organs, and targeting VEGF-A may result in a range of unwanted adverse events in other organ systems. Moreover, the effects of VEGF-A are very dosage-dependent and there is a narrow physiologic range (23-26), and thus, relatively small changes in VEGF-A may have a great effects. Indeed, studies have shown that inactivation of only one allele of VEGF-A in mice resulted in embryonic lethality at midgestation $(27,28)$, while a 2-fold increase in VEGF-A levels resulted in embryonic lethality in mice (29). Therefore, 

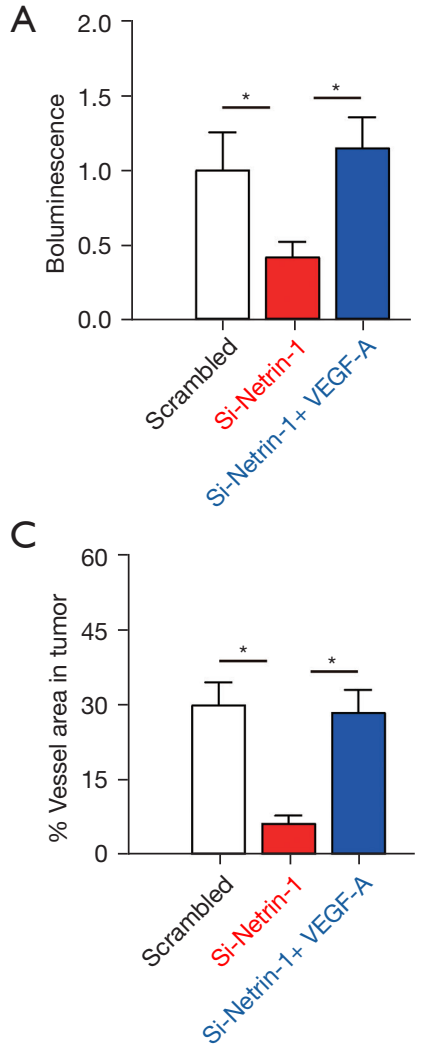

B
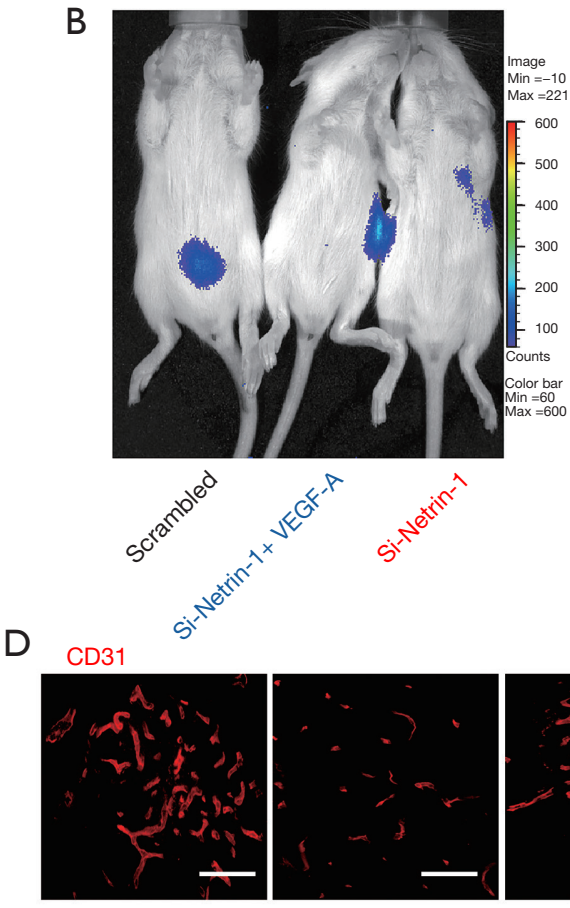

Scrambled

Si-Netrin-1

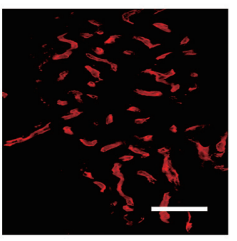

Si-Netrin-1+ VEGF-A

Figure 5 Silencing netrin-1 compromises $\mathrm{Rb}$ growth and tumor angiogenesis in vivo. Nude mice received subcutaneous transplantation of WERI-Rb-1 Rb cells transfected with either scrambled vector or si-netrin-1. (A,B) Rb tumor growth in vivo was assessed by bioluminescence; (C,D) tumor vessel density was analyzed by $\mathrm{CD} 31^{+}$area assessed by immunohistochemistry. *, $\mathrm{P}<0.05 ; \mathrm{N}=5(5$ mice in each experimental group); scale bar represents $100 \mu \mathrm{m}$. VEGF-A, vascular endothelial growth factor A.

targeting netrin-1 levels may be a much safer treatment in $\mathrm{Rb}$ patients. In addition, netrin-1 upregulation appears to be specific to $\mathrm{Rb}$ and other malignant disorders (30) and thus, any therapies which target netrin-1 may exert more specificity compared to therapies which target VEGF-A.

Inflammation and angiogenesis are often simultaneously coordinated in many pathological situations, and inflammation appears to be predisposing event for angiogenesis in diseases such as ischemic vascular diseases and cancers $(31,32)$. Therefore, future studies should investigate the effects of netrin-1 on $\mathrm{Rb}$-associated inflammation, specially, the effects on lymphocyte differentiation and recruitment, and macrophage infiltration and polarization.

\section{Acknowledgments}

Funding: This work was supported by the Natural Science
Foundation of China (No. 81901174 and No. 81873789).

\section{Footnote}

Reporting Checklist: The authors have completed the ARRIVE reporting checklist. Available at https://dx.doi. org/10.21037/atm-21-5560

Data Sharing Statement: Available at https://dx.doi. org/10.21037/atm-21-5560

Conflicts of Interest: All authors have completed the ICMJE uniform disclosure form (available at https://dx.doi. org/10.21037/atm-21-5560). The authors have no conflicts of interest to declare.

Ethical Statement: The authors are accountable for all aspects of the work in ensuring that questions related 


\section{Page 8 of 9}

to the accuracy or integrity of any part of the work are appropriately investigated and resolved. The study was conducted in accordance with the Declaration of Helsinki (as revised in 2013). All experiments were performed under a project license (SH9H-2019-A529-1) granted by the institutional ethics board of the Shanghai Jiao Tong University, in compliance with institutional guidelines for the care and use of animals.

Open Access Statement: This is an Open Access article distributed in accordance with the Creative Commons Attribution-NonCommercial-NoDerivs 4.0 International License (CC BY-NC-ND 4.0), which permits the noncommercial replication and distribution of the article with the strict proviso that no changes or edits are made and the original work is properly cited (including links to both the formal publication through the relevant DOI and the license). See: https://creativecommons.org/licenses/by-nc-nd/4.0/.

\section{References}

1. Kaewkhaw R, Rojanaporn D. Retinoblastoma: Etiology, Modeling, and Treatment. Cancers (Basel) 2020;12:2304.

2. Lee C, Kim JK. Chromatin regulators in retinoblastoma: Biological roles and therapeutic applications. J Cell Physiol 2021;236:2318-32.

3. Apte RS, Harbour JW. Inhibiting angiogenesis in retinoblastoma. Ophthalmic Res 2007;39:188-90.

4. Kvanta A, Steen B, Seregard S. Expression of vascular endothelial growth factor (VEGF) in retinoblastoma but not in posterior uveal melanoma. Exp Eye Res 1996;63:511-8.

5. Shokravi MT, Marcus DM, Alroy J, et al. Vitamin D inhibits angiogenesis in transgenic murine retinoblastoma. Invest Ophthalmol Vis Sci 1995;36:83-7.

6. Jockovich ME, Murray TG, Escalona-Benz E, et al. Anecortave acetate as single and adjuvant therapy in the treatment of retinal tumors of $\mathrm{LH}(\mathrm{BETA}) \mathrm{T}(\mathrm{AG})$ mice. Invest Ophthalmol Vis Sci 2006;47:1264-8.

7. Ferrara N. Vascular endothelial growth factor. Arterioscler Thromb Vasc Biol 2009;29:789-91.

8. Otrock ZK, Makarem JA, Shamseddine AI. Vascular endothelial growth factor family of ligands and receptors: review. Blood Cells Mol Dis 2007;38:258-68.

9. Murukesh N, Dive C, Jayson GC. Biomarkers of angiogenesis and their role in the development of VEGF inhibitors. Br J Cancer 2010;102:8-18.

10. Nikolova G, Lammert E. Interdependent development of
Yang et al. Netrin-1 promotes retinoblastoma-related angiogenesis

blood vessels and organs. Cell Tissue Res 2003;314:33-42.

11. Xiao X, Guo P, Chen Z, et al. Hypoglycemia reduces vascular endothelial growth factor A production by pancreatic beta cells as a regulator of beta cell mass. J Biol Chem 2013;288:8636-46.

12. Serafini T, Kennedy TE, Galko MJ, et al. The netrins define a family of axon outgrowth-promoting proteins homologous to C. elegans UNC-6. Cell 1994;78:409-24.

13. Bradford D, Cole SJ, Cooper HM. Netrin-1: diversity in development. Int J Biochem Cell Biol 2009;41:487-93.

14. Fitamant J, Guenebeaud C, Coissieux MM, et al. Netrin-1 expression confers a selective advantage for tumor cell survival in metastatic breast cancer. Proc Natl Acad Sci U S A 2008;105:4850-5.

15. Delloye-Bourgeois C, Fitamant J, Paradisi A, et al. Netrin-1 acts as a survival factor for aggressive neuroblastoma. J Exp Med 2009;206:833-47.

16. Arakawa $H$. Netrin-1 and its receptors in tumorigenesis. Nat Rev Cancer 2004;4:978-87.

17. Jiao X, Zhang D, Hong Q, et al. Netrin-1 works with UNC5B to regulate angiogenesis in diabetic kidney disease. Front Med 2020;14:293-304.

18. Barrett T, Wilhite SE, Ledoux P, et al. NCBI GEO: archive for functional genomics data sets--update. Nucleic Acids Res 2013;41:D991-5.

19. Zhou Y, Zhou B, Pache L, et al. Metascape provides a biologist-oriented resource for the analysis of systemslevel datasets. Nat Commun 2019;10:1523.

20. Wilson BD, Ii M, Park KW, et al. Netrins promote developmental and therapeutic angiogenesis. Science 2006;313:640-4.

21. Lu X, Le Noble F, Yuan L, et al. The netrin receptor UNC5B mediates guidance events controlling morphogenesis of the vascular system. Nature 2004;432:179-86.

22. Yang Y, Zou L, Wang Y, et al. Axon guidance cue Netrin-1 has dual function in angiogenesis. Cancer Biol Ther 2007;6:743-8.

23. Ferrara N, Gerber HP, LeCouter J. The biology of VEGF and its receptors. Nat Med 2003;9:669-76.

24. Carmeliet P, Jain RK. Molecular mechanisms and clinical applications of angiogenesis. Nature 2011;473:298-307.

25. Darland DC, Cain JT, Berosik MA, et al. Vascular endothelial growth factor (VEGF) isoform regulation of early forebrain development. Dev Biol 2011;358:9-22.

26. Wada T, Haigh JJ, Ema M, et al. Vascular endothelial growth factor directly inhibits primitive neural stem cell survival but promotes definitive neural stem cell survival. J 
Neurosci 2006;26:6803-12.

27. Carmeliet P, Ferreira V, Breier G, et al. Abnormal blood vessel development and lethality in embryos lacking a single VEGF allele. Nature 1996;380:435-9.

28. Ferrara N, Carver-Moore K, Chen H, et al. Heterozygous embryonic lethality induced by targeted inactivation of the VEGF gene. Nature 1996;380:439-42.

29. Miquerol L, Langille BL, Nagy A. Embryonic development is disrupted by modest increases in vascular endothelial growth factor gene expression. Development 2000;127:3941-6.

Cite this article as: Yang $\mathrm{X}$, Sun H, Tang T, Zhang W, Li Y. Netrin-1 promotes retinoblastoma-associated angiogenesis. Ann Transl Med 2021;9(22):1683. doi: 10.21037/atm-21-5560
30. Ylivinkka I, Keski-Oja J, Hyytiäinen M. Netrin-1: A regulator of cancer cell motility? Eur J Cell Biol 2016;95:513-20.

31. Mantovani A. Molecular pathways linking inflammation and cancer. Curr Mol Med 2010;10:369-73.

32. Porta C, Larghi P, Rimoldi M, et al. Cellular and molecular pathways linking inflammation and cancer. Immunobiology 2009;214:761-77.

(English Language Editor: J. Teoh) 